\title{
COMPLEX EXTREME MEASURABLE SELECTIONS
}

\author{
DOUGLAS MUPASIRI
}

(Received 15 July 1992)

Communicated by P. G. Dodds

\begin{abstract}
We give a characterization of complex extreme measurable selections for a suitable set-valued map. We use this result to obtain necessary and sufficient conditions for a function to be a complex extreme point of the closed unit ball of $L_{p}(\Omega, \Sigma, v ; X)$, where $(\Omega, \Sigma, v)$ is any positive, complete measure space, $X$ is a separable complex Banach space, and $0<p<\infty$.
\end{abstract}

1991 Mathematics subject classification (Amer. Math. Soc.): primary 28A05, 46E40.

\section{Introduction}

In 1969 Sundaresan [10] showed that sufficient conditions for a function $f$ to be an extreme point of the closed unit ball $L_{p}(\lambda, X)=L_{p}([0,1], \lambda, X)$, where $X$ is any Banach space, $\lambda$ is the Lebesgue measure on $[0,1]$, and $1<p<\infty$, are that $\|f\|_{p}=1$ and $f(t) /\|f(t)\|$ is an extreme point of the closed unit ball of $X$ for $\lambda$ almost all $t \in S_{f}:=\{s \in[0,1] \mid f(s) \neq 0\}$. In addition, Sundaresan showed that these conditions are also necessary if $X$ is finite dimensional. A year later, Sundaresan [11] proved that the same conditions characterize the extreme points of the closed unit ball of $L_{p}(\Omega, \Sigma, \mu ; X)$ if $X$ is a separable dual space, $\Omega$ is a locally compact Hausdorff space, and $\mu$ is a regular Borel measure. A significant improvement on Sundaresan's results was published 4 years later. In 1974 Johnson [7] used von Neumann's selection theorem to obtain a characterization of the extreme measurable selections for a suitably chosen set-valued map. As a corollary Johnson proved that if $X$ is a separable Banach space, $(\Omega, \Sigma, \mu)$ is any positive complete measure space, and $1<p<\infty$, then the so called natural conditions characterize the extreme points of the closed unit ball of $L_{p}(\Omega, \Sigma, \mu ; X)$, thus extending considerably Sundaresan's results. Johnson also gave necessary and sufficient conditions for a function to be an

(C) 1995 Australian Mathematical Society 0263-6115/95 \$A2.00+0.00 
extreme point of $L_{\infty}(\Omega, \Sigma, \mu ; X)$ in the special case where $\Omega$ is a Polish space.

In a very real sense Johnson's result is the best possible. In 1982 Greim [6] constructed an example of a non-separable Banach space $X$ and a function $f$ on $[0,1]$ which is an extreme point of the closed unit ball of all $L_{p}(\lambda, X)$ spaces, $1<p<\infty$, and for which no value $f(t)$ is an extreme point of the closed ball of $X$ of radius $\|f(t)\|$. This shows that, in general, the separability assumption on $X$ in Johnson's result cannot be dropped. We also note in this connection that Johnson's characterization fails if $p=1$. To see this observe that the function $f=\chi_{[0,1]}$ (the characteristic function of $[0,1])$ satisfies the natural conditions but is not an extreme point of $L_{1}(\lambda)=L_{1}(\lambda, \mathbb{R})$.

In this paper we consider complex extreme points, the complex analogues of (real) extreme points. We prove that the complex extreme measurable selections for a suitable set-valued map admit a characterization which parallels the one given by Johnson for (real) extreme measurable selections for the same set-valued map. We then use this result to show that if $X$ is a separable complex Banach space, $(\Omega, \Sigma, v)$ is any positive, complete measure space and $0<p<\infty$ (we consider the case $p=\infty$ separately), then the natural conditions characterize the complex extreme points of $B_{L_{p}(\Omega, \Sigma, \nu ; X)}$. Here too, Greim's example can be appropriately modified to show that our result may also fail if $X$ is not separable.

This paper constitutes part of the author's Ph.D. dissertation which will be submitted to Northern Illinois University.

\section{Preliminaries}

Let $(Y,\|\cdot\|)$ be any quasi-normed space, and let $B_{Y}$ denote the closed unit ball of $Y$, that is, $B_{Y}=\{y \in Y:\|y\| \leq 1\}$. Also, if $X$ is any Banach space, $(\Omega, \Sigma, \nu)$ any positive measure space and $0<p \leq \infty, L_{p}(\mu, X)=L_{p}(\Omega, \Sigma, \mu ; X)$ is the LebesgueBochner space of $\mu$-equivalence classes of strongly measurable functions $f: \Omega \rightarrow X$ for which $\int_{\Omega}\|f(\omega)\|^{p} d \mu(\omega)<\infty$ if $0<p<\infty$ and ess $\sup _{\omega \in \Omega}\|f(\omega)\|<\infty$ if $p=\infty$, quasi-normed by

$$
\|f\|_{p}=\left\{\begin{array}{lll}
{\left[\int_{\Omega}\|f(\omega)\|^{p} d \mu(\omega)\right]^{\frac{1}{p}}} & \text { if } & 0<p<\infty \\
\operatorname{ess~sup}_{\omega \in \Omega}\|f(\omega)\| & \text { if } & p=\infty
\end{array}\right.
$$

DEFINITION 0.1. A point $x$ of a (not necessarily convex) subset $K$ of a complex vector space $V$ is called a complex extreme point of $K$ if and only if $\{x+z y:|z| \leq$ 1) $\subset K$ for $y \in V$ implies $y=0$. We denote the set of complex extreme points of $K$ by c-ext $K$. If $K$ is convex, then $x \in$ c-ext $K$ if and only if $\{x+z y:|z|=1\} \subset K$ for $y \in V$ implies $y=0$ if and only if $\{x+z y: z= \pm 1, \pm i\} \subset K$ for $y \in V$ implies $y=0$. Note that every (real) extreme point of a convex set $K$ is a complex extreme point of $K$. 
DEFINITION 0.2. Let $(\Omega, \Sigma, \mu)$ be any measure space (we assume that all our measures are positive). The outer measure induced by $\mu$ is the function $\mu^{*}: \mathscr{P}(\Omega) \rightarrow$ $[0, \infty]$ defined by

$$
\mu^{*}(A)=\inf \{\mu(B): B \in \Sigma, A \subset B\}
$$

for all $A \in \mathscr{P}(\Omega)$ where $\mathscr{P}(\Omega)$ is the power set of $\Omega$. A set $C \subset \Omega$ is $\mu^{*}$-measurable if and only if

$$
\mu^{*}(A)=\mu^{*}(A \cap C)+\mu^{*}\left(A \cap C^{c}\right)
$$

for each $A \subset \Omega$.

Let $\mathscr{M}_{\mu^{*}}$ denote the $\sigma$-algebra of all $\mu^{*}$-measurable subsets of $\Omega$. Then the restriction of $\mu^{*}$ to $\mathscr{M}_{\mu^{*}}$ is a complete measure. If we let $\Sigma_{\mu}$ be the $\mu$-completion of $\Sigma$, so that $\Sigma_{\mu}:=\left\{A \subset \Omega\right.$ : there exist $A_{1}, A_{2} \in \Sigma$ with $A_{1} \subset A \subset A_{2}$ and $\mu\left(A_{2} \backslash A_{1}\right)=0$, then $\Sigma_{\mu}$ is a sub- $\sigma$-algebra of $\mathscr{M}_{\mu^{*}}$. If $\mu$ is $\sigma$-finite, then $\Sigma_{\mu}=\mathscr{M}_{\mu^{*}}$. We deduce from this fact that if $\Omega$ is a Polish space and $\mu$ is $\sigma$-finite, then the restriction of $\mu^{*}$ to $\Sigma_{\mu}$ is a regular measure. (See [2, Proposition 8.1.10]).

DEFINITION 0.3. Let $(\Omega, \Sigma, \mu)$ be a measure space and let $X$ be a Hausdorff space. A function $f: \Sigma \rightarrow X$ is $\Sigma$-measurable if and only if the following conditions hold:

(i) $f^{-1}(G) \in \Sigma$ for every open set $G \subset X$;

(ii) $f$ is $\mu$-essentially separably valued, that is, there exists $N \in \Sigma$ with $\mu(N)=0$ such that $f(\Omega \backslash N)$ is a separable subset of $X$.

If $\Sigma=\mathscr{M}_{\mu^{*}}$ and $f$ satisfies the two conditions above, then we shall simply say $f$ is $\mu$-measurable. By a similar abuse of notation we shall refer to the elements of $\mathscr{M}_{\mu^{*}}$ as the $\mu$-measurable subsets of $\Omega$; indeed we shall often identify $\mu$ with the restriction of $\mu^{*}$ to $\mathscr{M}_{\mu^{*}}$.

DefintTion 0.4. A subset of a metric space is called analytic (Souslin) if it is a continuous image of a Borel subset of some Polish (that is, complete separable metric) space.

Let $2^{S}$ denote the power set of a set $S$. The graph of a set-valued map $F: T \rightarrow 2^{S}$ is the set

$$
\mathscr{G}_{F}:=\{(t, s) \in T \times S \mid s \in F(t)\} .
$$

A set-valued map is said to be Borel measurable (respectively, analytic) if and only if its graph is a Borel set (respectively, an analytic set).

In the sequel we shall need to use von Neumann's measurable selection theorem [12] and a corollary of the theorem due to Aumann [1]. We quote below the form of these results given in [7] as this form is more suitable for our needs. 
THEOREM 0.5. (von Neumann) Let $M$ and $N$ be Polish spaces, let $A \subset M$ be an analytic set and $g: A \rightarrow N$ be a continuous function. Suppose $\mu$ is a Borel measure on $N$. Then the set $g(A)$ is $\mu$-measurable and there exists a $\mu$-measurable map $\Phi: g(A) \rightarrow M$ such that $g(\Phi(x))=x$ for all $x$ in $g(A)$.

COROLlaRY 0.6. (Aumann) Let $S_{1}$ and $S_{2}$ be Polish spaces and let $F: S_{1} \rightarrow 2^{S_{2}}$ be an analytic map such that $F(s) \neq \emptyset$ for all $s \in S_{1}$. Suppose $\mu$ is a Borel measure on $S_{1}$. Then there exists a $\mu$-measurable function $f: S_{1} \rightarrow S_{2}$ with $f(s) \in F(s)$ for all $s \in S_{1}$.

For the remainder of this paper we shall fix the following notation. Unless specified otherwise, $X$ shall be a separable complex Banach space, $S$ shall be a Polish space and $\mu$ shall be a positive, $\sigma$-finite Borel, and hence regular measure on $S$ [2].

As in [7], $\tilde{A}=A^{c}$ denotes the complement of a set $A, B \sim A:=B \cap \tilde{A}$ is the complement of $A$ in $B, A+B=\{x+y \mid x \in A, y \in B\}$ and $A-B=\{x-y \mid x \in$ $A, y \in B\}$.

\section{The Main Result}

LEMMA 1.1. (Johnson [7]) Let $F, G: S \rightarrow 2^{X}$ be Borel measurable maps. Then the maps $H_{i}, 1 \leq i \leq 5$, defined below are Borel measurable.

(1) $H_{1}(s)=F(s) \cap G(s)$.

(2) If $A \subset S$ is a Borel set, $H_{2}(s)=F(s)$ if $s \in A$, and $H_{2}(s)=G(s)$ if $S \in \tilde{A}$.

(3) If $f: S \rightarrow X$ is Borel measurable and $\lambda \in \mathbb{C}, H_{3}(s)=f(s)+\lambda F(s)$.

(4) If $B \subset X$ is a Borel set, $H_{4}(s)=B$ for all $s \in S$.

(5) $H_{5}(s)=F(s) \times G(s)$, where $(F(s) \times G(s):=\{(x, y) \mid x \in F(s), y \in G(s)\})$.

We now prove our main result. We remark that in what follows we shall always identify functions which are equal almost everywhere with respect to a given measure.

THEOREM 1.2. Let $F: S \rightarrow 2^{x}$ be a Borel measurable with $F(s) \neq \emptyset$ and convex for all $s \in S$. Define $F_{1}: S \rightarrow 2^{X}$ by $F_{1}(s)=F(s) \sim$ c-ext $F(s)$ and suppose that $\left\{s \in S \mid F_{1}(s) \neq \emptyset\right\}$ is a Borel set. Let $F_{F}$ denote the set of all $\mu$-measurable functions $f: S \rightarrow X$ such that $f(s) \in F(s)$ for $\mu$-almost all $s \in S$ (such functions are called measurable selections for $F$ ). Then $f \in \mathrm{c}$-ext $F_{F}$ if and only if $f(s) \in \mathrm{c}$-ext $F(s)$ for $\mu$-almost all $s \in S$.

PROOF. Let $A=\left\{s \in S \mid f(s) \in F_{1}(s)\right\}$ and suppose that $A$ is not of $\mu$-measure zero. Define $H: S \rightarrow 2^{X^{4}}\left(X^{4}=X \times X \times X \times X\right)$ by

$$
H(s)=\left\{\begin{array}{lll}
F(s) \times F(s) \times F(s) \times F(s) & \text { if } & s \in S \sim F_{1}^{-1}(\emptyset), \\
\{0\} \times\{0\} \times\{0\} \times\{0\} & \text { if } & S \in F_{1}^{-1}(\emptyset) .
\end{array}\right.
$$


Then $H$ is a Borel measurable map by Lemma 1.1. Let $h: S \times X \times X \rightarrow S \times X^{4}$ be the map $(s, x, y) \mapsto(s, x+y, x-y, x+i y, x-i y)$. Then the set

$$
U:=h^{-1}\left(\mathscr{G}_{H}\right) \cap\left[\left[S \sim F_{1}^{-1}(\emptyset)\right] \times X \times[X \sim\{0\}]\right]
$$

is a Borel set. Moreover, if $\Phi: S \times X \times X \rightarrow S \times X$ is the map $(s, x, y) \mapsto(s, x)$, then $\Phi(U)=\mathscr{G}_{F_{1}}$. Hence $\mathscr{G}_{F_{1}}$ is analytic. By [5, Propositions 13 and 14, p. 97] and the regularity of the measure $\mu$, there exists a Borel measurable function $g: S \rightarrow X$ such that $g=f \mu$-almost everywhere (henceforth abbreviated $\mu$-a.e.).

Let $B:=\left\{s \in S \mid g(s) \in F_{1}(s)\right\}$ and let $\Pi_{1}$ be the canonical projection of $S \times X$ onto $S$. Then $\Pi_{1}\left(\mathscr{G}_{g} \cap \mathscr{G}_{F_{1}}\right)=B$, so $B$ is analytic and hence $\mu$-measurable by Theorem 0.5. (Note that the family of analytic subsets of a Polish space is closed under countable intersections, countable unions and countable products - (see $[8, \mathrm{p}$. 435] or [2, Propositions 8.2.2, p. 261 and 8.2.5, p. 263]).

Since $g=f \mu$-a.e., we must have $\mu(B)>0$, otherwise $\mu(A)=0$, a contradiction. By the regularity of $\mu$ there exists a compact set $K \subset B$ with $\mu(K)>0$. Let $G: S \rightarrow 2^{X}$ be defined by the expression

$$
[(-g(s)+F(s)) \cap(g(s)-F(s)) \cap i(-g(s)+F(s)) \cap i(g(s)-F(s))] \sim\{0\}
$$

if $s \in K$ and let $G(s)=\{0\}$ if $s \in S \sim K$. Then $G$ is Borel measurable by Lemma 1.1. Also, $G(s) \neq \emptyset$ for $s \in S$. Hence by Corollary 0.6 there exists a $\mu$-measurable function $h_{1}: S \rightarrow X$ such that $h_{1}(s) \in G(s)$ for all $s \in S$. Note that $h_{1}(s) \neq 0$ for all $s \in K$ by definition of $G$. Moreover $\left\{g(s)+z h_{1}(s) \mid z= \pm 1, \pm i\right\} \subset F(s)$ for all $s \in K$, and since $g=f \mu$-a.e., $\left\{g(s)+z h_{1}(s) \mid z= \pm 1, \pm i\right\}=\{g(s)\} \subset F(s)$ for $\mu$-almost all $s \in S \sim K$. Hence $\left\{g(s)+z h_{1}(s) \mid z= \pm 1, \pm i\right\} \subset F(s)$ for $\mu$-almost all $s \in S$, so $g \notin$ c-ext $F_{F}$. Since $f=g \mu$-a.e., $f \notin$ c-ext ${ }_{F}$. This completes the proof of the necessity of the condition. The proof of the converse is immediate.

COROLLARY 1.3. If $K$ is a non-empty, convex Borel subset of $X$ and ${ }_{K}:=\{f$ : $S \rightarrow K \mid f$ is $\mu$-measurable $\}$, then $f \in \mathrm{c}$-ext $\mathcal{W}_{K}$ if and only if $f(s) \in \mathrm{c}$-ext $K$ for $\mu$-almost all $s \in S$.

The proof of this corollary is straightforward, and is therefore omitted. As a consequence of Corollary 1.3, we get the following characterization of the complex extreme points of $\mathscr{B}_{L_{\infty}(\mu, X)}:$ a function $f \in \mathrm{c}$-ext $B_{L_{\infty}(\mu, X)}$ if and only if $f(s) \in$ c-ext $B_{X}$ for $\mu$-almost all $s \in S$.

COROLlARY 1.4. Let $K$ be a non-empty, closed, convex subset of $X$ and let $v$ be any positive $\sigma$-finite Borel measure on $K$. If $v(K \sim \mathrm{c}$-ext $K)>0$, then there exists $a$ Borel measurable function $g: K \rightarrow X$ such that $g \neq 0$ on a set of positive $\nu$-measure and such that $\{x+z g(x) \mid z= \pm 1, \pm i\} \subset K$ for v-almost all $x \in K$. 
PRoOF. Let $S=K$ in Corollary 1.3 and define $F: K \rightarrow 2^{E}$ by $F(x)=K$ for all $x \in K$ and put $F_{1}(x)=F(x) \sim$ c-ext $F(x)=K \sim$ c-ext $K$ for all $x \in K$. Then by an argument similar to the one used in the proof of Theorem 1.2, it is easy to see that $K \sim$ c-ext $K$ is analytic and hence $\nu$-measurable. Note that by Corollary 1.3, id $_{K}$ (the identity map on $K$ ) is not a complex extreme point of the set of functions defined by $\mathcal{F}_{K}:=\{f: K \rightarrow K \mid f$ is $\nu$-measurable $\}$. For if id $\operatorname{id}_{K} \in \mathrm{c}$-ext ${ }_{K}$, then $x=\operatorname{id}_{K}(x) \in$ c-ext $K$ for $v$-almost all $x$ in $K$, contradicting $v(K \sim$ c-ext $K)>0$. Thus there exists a $\nu$-measurable function $g_{0}: K \rightarrow X$ such that $g_{0} \neq 0$ on a set of positive $\nu$-measure and $\left\{\mathrm{id}_{K}+z g_{0} \mid z= \pm 1, \pm i\right\} \subset \mathbb{B}_{K}$, that is,

$$
\left\{x+z g_{0}(x) \mid z= \pm 1, \pm i\right\}=\left\{\operatorname{id}_{K}(x)+z g_{0}(x) \mid z= \pm 1, \pm i\right\} \subset K
$$

for $v$-almost all $x \in K$. As in the proof of Theorem 1.2 we appeal to [5, p.97, Proposition 13 and 14] and use the regularity of $v$ to get a Borel measurable function $g: K \rightarrow X$ such that $g=g_{0} \nu$-a.e. It is now clear that $\{x+z g(x) \mid z= \pm 1, \pm i\} \subset K$ for $\nu$-almost all $x \in K$ as desired.

In the proof of the next corollary we shall need the following:

DEFINITION 1.5. Let $Y$ be any Polish space and $\mathscr{B}(Y)$ be the Borel subsets of $Y$. For each positive Borel measure $\nu$, let $\nu^{*}$ be the outer measure induced by $\nu$. Let $\mathscr{M}_{v^{*}}$ be the $\sigma$-algebra of $v^{*}$-measurable subset of $Y$. Then the universally measurable subsets of $Y$ are the elements of the $\sigma$-algebra

$$
\mathscr{U}(\mathscr{B}(Y)):=\bigcap\left\{\mathscr{M}_{v^{*}}: v \text { is a finite Borel measure on } Y\right\} .
$$

Since analytic subsets of a Polish space $Y$ are measurable with respect to every Borel measure on $Y$ (Theorem 0.5 ), we deduce that analytic subsets are universally measurable.

COROLLARY 1.6. Let $K$ be a non-empty, closed, convex subset of $X$ and let $(\Omega, \Sigma, \nu)$ be any $\sigma$-finite positive, complete measure space. Let $:=\{f: \Omega \rightarrow K \mid f$ is $\Sigma$-measurable, that is, $f^{-1}(B) \in \Sigma$ for all $\left.B \in \mathscr{B}(K)\right\}$ (here $\mathscr{B}(K)$ denotes the Borel subsets of $K$ ). Then $f \in \mathrm{c}$-ext if and only if $f(w) \in \mathrm{c}$-ext $K$ for $v$-almost all $\omega \in \Omega$.

PROOF. Suppose $f \in$ c-ext and let $\mu: \mathscr{B}(K) \rightarrow[0, \infty]$ be the image measure of $\nu$ under $f$, that is, $\mu(B)=\nu\left(f^{-1}(B)\right)$ for all $B \in \mathscr{B}(K)$. Then $\mu$ is a Borel measure on $K$. But $\mu$ need not be $\sigma$-finite. Indeed, if $x_{0} \in K$ and $f$ is the constant function $f(\omega)=x_{0}$ for all $\omega \in \Omega$, then $\mu$ is not $\sigma$-finite unless $\nu(\Omega)<\infty$. However, $\mu$ has the nice property that the restriction of the outer measure $\mu^{*}$ (induced by $\mu$ ) to 
$\mathscr{U}(\mathscr{B}(K))$ is a regular measure. This means, in particular, that $\mu^{*}$ is regular on the analytic subsets of $K$. Thus, mutatis mutandis, Theorem 1.2 remains valid for this measure.

Suppose the set $A=\{\omega \in \Omega \mid f(\omega) \notin$ c-ext $K\}$ is not of $\nu$-measure zero. We may assume that $f \neq 0$ on a set positive $\nu$-measure. As in Corollary 1.3, $K \sim$ c-ext $K$ is analytic and hence $\mu$-measurable. If $\mu(K \sim$ c-ext $K)=0$, then by the outer regularity of $\mu^{*}$ on $\mathscr{U}(\mathscr{B}(K))$ there exists a $G_{\delta}$-set $B$ such that $(K \sim$ c-ext $K) \subset B \subset K$ and $\mu(B)=0$. Now $A \subset f^{-1}(B)$ and $v\left(f^{-1}(B)\right)=\mu(B)=0$ and so, by the completeness of $(\Omega, \Sigma, v), v(A)=0$, a contradiction. Therefore $\mu(K \sim$ c-ext $K)>0$. Thus by Corollary 1.4 , there is a Borel measurable function $g: K \rightarrow X$ such that $g \neq 0$ on a set of positive $\mu$-measure and $\{x+z g(x) \mid z=$ $\pm 1, \pm i\} \subset K$ for $\mu$-almost all $x \in K$. Evidently $0<\mu\left(S_{g}\right)=\nu\left(f^{-1}\left(S_{g}\right)\right)$ and $(g \circ f)(\omega)=g(f(\omega)) \neq 0$ for all $\omega \in f^{-1}\left(S_{g}\right)$. Thus $g \circ f: S \rightarrow X$ is a $\nu$ measurable function which never vanishes on the set $f^{-1}\left(S_{g}\right)$ of positive $v$-measure. Moreover, by our choice of the function $g,\{f(\omega)+z(g \circ f)(\omega) \mid z= \pm 1, \pm i\} \subset K$ for $\nu$-almost all $\omega \in \Omega$, that is, $\{f+z(g \circ f) \mid z= \pm 1, \pm i\} \subset$. Hence $f \notin \mathrm{c}$-ext This completes the proof of the necessity of the condition. The proof of the sufficiency of the condition is immediate.

\section{An application}

THEOREM 2.1. Let $(\Omega, \Sigma, v)$ be any positive, complete measure space. Let $f \in$ $L_{p}(\nu, X)=L_{p}(\Omega, \Sigma, \nu ; X), 0<p<\infty$. Then $f \in \mathrm{c}$-ext $B_{L_{p}(\nu, X)}$ if and only if $\|f\|_{p}=1$ and $f(\omega) /\|f(\omega)\| \in \mathrm{c}$-ext $B_{X}$ for $\nu$-almost all $\omega \in S_{f}$.

PROOF. Let $f \in$ c-ext $B_{L_{p}(v, X)}$. Then clearly $\|f\|_{p}=1$. Let $\Sigma_{S_{f}}:=\{A \subset$ $\left.S_{f} \mid A \in \Sigma\right\}=\left\{B \subset \Omega \mid B=A \cap S_{f}, A \in \Sigma\right\}$. Then $\left(S_{f}, \Sigma_{f}, v\right)$ is a positive, complete, $\sigma$-finite measure space. Define $h: S_{f} \rightarrow B_{X}$ by $h(\omega)=f(\omega) /\|f(\omega)\|$. Put $:=\left\{v: S_{f} \rightarrow B_{X} \mid v\right.$ is $\Sigma_{f}$-measurable $\}$. If $h \notin \mathrm{c}$-ext then there exists $g: S_{f} \rightarrow X$ such that $g \neq 0$ on a set of positive $v$-measure and $\{h+z g:|z| \leq 1\} \subset$. Hence $\{h(\omega)+z g(\omega):|z| \leq 1\}=\{f(\omega) /\|f(\omega)\|+z g(\omega):|z| \leq 1\} \subset B_{X}$ for $\nu$-almost all $S \in S_{f}$. Let $h_{1}: \Omega \rightarrow X$ be given by

$$
h_{1}(\omega)=\left\{\begin{array}{lll}
\|f(\omega)\| g(\omega) & \text { if } & \omega \in S_{f}, \\
0 & \text { if } & \omega \in \Omega \sim S_{f}
\end{array}\right.
$$

Then, since $v\left(S_{g}\right)>0$ and $S_{h_{1}}=S_{g}$, it follows that $h_{1} \neq 0$ on a set of positive $v$-measure. Moreover,

$$
\left\{f(\omega)+z h_{1}(\omega):|z| \leq 1\right\}=\{f(\omega)+z\|f(\omega)\| g(\omega):|z| \leq 1\} \subset\|f(\omega)\| B_{X}
$$


for $\nu$-almost all $\omega \in S_{f}$, so $\left\|f(\omega)+z h_{1}(\omega)\right\| \leq\|f(\omega)\|$ for all $z \in \mathbb{C}$ with $|z| \leq 1$ and for $\nu$-almost all $\omega \in S_{f}$. Since $h_{1}(\omega)=0$ if $\omega \in \Omega \sim S_{f}$, we have $\| f\left(\omega+z h_{1}(\omega) \| \leq\right.$ $\|f(\omega)\|$ for all $z \in \mathbb{C}$ with $|z| \leq 1$ and for $\nu$-almost all $\omega \in \Omega$. Consequently, $\left\|f+z h_{1}\right\|_{p} \leq\|f\|_{p}=1$ for all $z \in \mathbb{C}$ with $|z| \leq 1$ or equivalently $\left\{f+z h_{1}:|z| \leq\right.$ 1) $\subset B_{L_{p}(v, X)}$. Hence, $f \notin$ c-ext $B_{L_{p}(v, X)}$, a contradiction. Therefore we must have $h \in$ c-ext . We now apply Corollary 1.6 to the positive, complete, $\sigma$-finite measure space $\left(S_{f}, \Sigma_{f}, v\right)$, the $\Sigma_{S_{f}}$-measurable function $h$, and the non-empty, closed, convex set $B_{X}$, to get that $h(\omega) \in$ c-ext $B_{X}$ for $\nu$-almost all $\omega \in S_{f}$. This proves the necessity of the condition.

The proof of the converse is essentially contained in Theorem 2.5 of [4]. Suppose $\|f\|_{p}=1$ and $f(\omega) /\|f(\omega)\| \in$ c-ext $B_{X}$ for $\nu$-almost all $\omega \in S_{f}$. Let $0 \neq g \in$ $L_{p}(\nu, X)$. Then the set $B=\{\omega \in \Omega \mid g(\omega) \neq 0\}$ has positive $v$-measure and for $\nu$-almost all $\omega \in B$ there exists $\theta_{\omega}$ with $0 \leq \theta_{\omega} \leq 2 \pi$ such that $\left\|f(\omega)+\mathrm{e}^{i \theta_{\omega}} g(\omega)\right\|>$ $\|f(\omega)\|$. For if $\omega \in B \sim S_{f}$, then $\left\|f(\omega)+\mathrm{e}^{i \theta} g(\omega)\right\|=\|g(\omega)\|>0=\|f(\omega)\|$ for all $\theta, 0 \leq \theta \leq 2 \pi$; and if $\omega \in B \cap S_{f}^{0}$, where $S_{f}^{0}:=\left\{\tau \in S_{f}: f(\tau) /\|f(\tau)\| \in\right.$ c-ext $\left.B_{X}\right\}$, then the assertion follows from the definition of c-ext $B_{X}$.

Claim. Let $N \subset B$ be the exceptional set in the preceding assertion, that is, $N=\left\{\omega \in B:\left\|f(\omega)+\mathrm{e}^{i \theta} g(\omega)\right\| \leq\|f(\omega)\|\right.$ for all $\left.\theta, 0 \leq \theta \leq 2 \pi\right\}$. Then

$$
\int_{0}^{2 \pi}\left[\left\|f(\omega)+\mathrm{e}^{i \theta} g(\omega)\right\|^{p}-\|f(\omega)\|^{p}\right] \frac{\mathrm{d} \theta}{2 \pi}>0
$$

for each $\omega \in B \sim N$.

\section{PROOF OF Claim.}

CASE 1: $\|g(\omega)\| \leq \| f(\omega \|$. In this case, the claim follows from the proof of (b) implies (d) in [4, Proposition 2.2].

CAse 2: $\|g(\omega)\|>\|f(\omega)\|$. Since every normed linear space is locally PL-convex (see [3] for the definition) it follows from [3, Proposition 2.2] that $\log \|\cdot\|$ is a plurisubharmonic function on $X$, that is,

$$
\log \|x\| \leq \int_{0}^{2 \pi} \log \left\|x+\mathrm{e}^{i \theta} y\right\| \frac{\mathrm{d} \theta}{2 \pi} \quad \text { for all } \quad x, y \in X
$$

Hence $\|\cdot\|^{p}$ is plurisubharmonic on $X$. We therefore have for all $\omega \in B \sim N$

$$
\begin{aligned}
\int_{0}^{2 \pi}\left\|f(\omega)+\mathrm{e}^{i \theta} g(\omega)\right\|^{p} \frac{\mathrm{d} \theta}{2 \pi} & =\int_{0}^{2 \pi}\left\|g(\omega)+\mathrm{e}^{i \theta} f(\omega)\right\|^{p} \frac{\mathrm{d} \theta}{2 \pi} \\
& \geq\|g(\omega)\|^{p}>\|f(\omega)\|^{p}
\end{aligned}
$$


or equivalently, for all $\omega \in B \sim N$

$$
\int_{0}^{2 \pi}\left[\left\|f(\omega)+\mathrm{e}^{i \theta} g(\omega)\right\|^{p}-\|f(\omega)\|^{p}\right] \frac{\mathrm{d} \theta}{2 \pi}>0
$$

as claimed. It now follows readily from Fubini's Theorem that

$$
\int_{0}^{2 \pi} \int_{\Omega} \chi_{b}(\omega)\left[\left\|f(\omega)+\mathrm{e}^{i \theta} g(\omega)\right\|^{p}-\|f(\omega)\|^{p}\right] \mathrm{d} \nu(\omega) \frac{\mathrm{d} \theta}{2 \pi}>0 .
$$

So

$$
\begin{aligned}
\int_{0}^{2 \pi} & \left\|f+\mathrm{e}^{i \theta} g\right\|_{L_{p}(v, X)}^{p} \frac{\mathrm{d} \theta}{2 \pi}-\|f\|_{L_{p}(v, X)}^{p} \\
= & \int_{0}^{2 \pi} \int_{\Omega} \chi_{b}(\omega)\left[\left\|f(\omega)+\mathrm{e}^{i \theta} g(\omega)\right\|^{p}-\|f(\omega)\|^{p}\right] \mathrm{d} \nu(\omega) \frac{\mathrm{d} \theta}{2 \pi} \\
& \quad+\int_{0}^{2 \pi} \int_{\Omega \backslash B}\left[\|f(\omega)\|^{p}-\|f(\omega)\|^{p}\right] \mathrm{d} \nu(\omega) \frac{\mathrm{d} \theta}{2 \pi} \\
= & \int_{0}^{2 \pi} \int_{\Omega} \chi_{b}(\omega)\left[\left\|f(\omega)+\mathrm{e}^{i \theta} g(\omega)\right\|^{p}-\|f(\omega)\|^{p}\right] \mathrm{d} \nu(\omega) \frac{\mathrm{d} \theta}{2 \pi}>0 .
\end{aligned}
$$

Thus,

$$
\int_{0}^{2 \pi}\left\|f+\mathrm{e}^{i \theta} g\right\|_{L_{p}(v, X)}^{p} \frac{\mathrm{d} \theta}{2 \pi}>1 .
$$

Therefore there exists $\theta_{0}, 0 \leq \theta_{0} \leq 2 \pi$ such that $\left\|f+\mathrm{e}^{i \theta_{0}} g\right\|_{L_{p}(v, X)}^{p}>1$. Hence $f \in \mathrm{c}$-ext $B L_{p}(\nu, X)$, as desired.

COROLLARY 2.2. Let $X$ be a separable complex Banach space. Then $x=\left(x_{n}\right)_{n=1}^{\infty} \subset$ $B_{\ell_{1}(X)}$ is a complex extreme point of $B_{\ell_{1}(X)}$ if and only if $\|x\|_{\ell_{1}(X)}:=\sum_{n=1}^{\infty}\left\|x_{n}\right\|=1$ and, for each non-zero coordinate $x_{n}$, the point $x_{n} /\left\|x_{n}\right\|$ is a complex extreme point of $B_{X}$.

REMARK 2.3. The characterization of the complex extreme points of $B_{\ell_{1}(X)}$ given in Corollary 2.2 is (except for the requirement that $X$ be a separable complex Banach space) of the same form as the characterization of the (real) extreme points of real $\ell_{p}(X)$ for $1<p<\infty$ [9]. By contrast, an element $x$ in the real $\ell_{1}(X)$ is an extreme point of the $B_{\ell_{1}(X)}$ if and only if $\|x\|_{\ell_{1}(X)}=1$ and $x$ has the form $x=\left(0,0,0, \cdots, 0, x_{n}, 0, \ldots\right)$ where $x_{n}$ is a real extreme point of $B_{X}$.

Finally, we note that since the notion of complex extreme points makes sense even in non-convex settings, a natural question to ask is whether Theorem 2.1 holds if 
we replace the Banach space $X$ by a separable complex continuously quasi-normed space. We have so far been unable to answer this question. What is clear, however, is that our method of proof does not extend to this more general situation because the method depends on the Hahn-Banach Theorem.

\section{Acknowledgments}

The author wishes to thank Professors Patrick N. Dowling and Mark A. Smith of Miami University of Ohio (where this work was done in the spring of 1991) for inviting him to Miami University and for their helpful comments and suggestions. He also thanks the Mathematics/Statistics Department secretary, Jean Cavalieri, for a singularly excellent job of typing the paper. Lastly, but not least, the author wishes to extend special thanks to his dissertation advisor, Professor Robert F. Wheeler of Northern Illinois University, for his advice, encouragement and support.

\section{References}

[1] R. J. Aumann, 'Integrals of set-valued functions', J. Math. Anal. Appl. 12 (1965), 1-12.

[2] D. L. Cohn, Measure theory (Birkhäuser, Boston, 1980).

[3] W. J. Davis, D. J. H. Garling and N. Tomzcak-Jaegermann, 'The complex convexity of quasinormed spaces', J. Funct. Anal. 55 (1984), 110-150.

[4] S. J. Dilworth, 'Complex convexity and the geometry of Banach spaces', Math. Proc. Cambridge Philos. Soc. 99 (1986), 495.

[5] N. Dinculeanu, Vector measures, Internat. Ser. Monographs Pure Appl. Math. 95 (Pergamon Press, Oxford, 1967).

[6] P. Greim, 'An extremal vector-valued $l^{p}$-function taking no extremal vectors as values', Proc. Amer. Math. Soc. 84 (1982), 65-68.

[7] J. A. Johnson, 'Extreme measurable selections', Proc. Amer. Math. Soc. 44 (1974), 107-112.

[8] K. Kuratowski and A. Mostowski, Set theory, Stud. Logic Found. Math. (North-Holland, Amsterdam, 1976).

[9] M. A. Smith, Rotundity and extremity in $l_{p}\left(x_{i}\right)$ and $l^{p}(\mu, x)$, Contemp. Math. 52 (Amer. Math. Soc., Providence, 1980) pp. 143-162.

[10] K. Sundaresan, 'Extreme points of the unit cell in Lebesgue-Bochner function spaces. I', Proc. Amer. Math. Soc. 23 (1969), 179-184.

[11] _ 'Extreme points of the unit cell in Lebesgue-Bochner function spaces', Colloq. Math. 22 (1970), 111-119.

[12] J. von Neumann, 'On rings of operators. Reduction theory', Ann. of Math. (2) 50 (1949), 401-485.

\section{Current Address}

Department of Mathematics and Statistics

Miami University

Oxford, Ohio 45056
Permanent Address

Department of Mathematics

University of Northern Iowa

Cedar Falls, Iowa 50614-0506 